\title{
GIANT SOFT TISSUE SARCOMA OF SCALP WITH SKULL AND GEREBRAL INVASION
}

\author{
Raghav Yelamanchi, * Parikshith Manjunath, Nikhil Gupta, CK Durga
}

Department of Surgery, Atal Bihari Vajpayee Institute of Medical Sciences and Dr. Ram Manohar Lohia Hospital, New Delhi, India, Pin-110001

\section{ABSTRACT}

Scalp soft tissue sarcomas (STS) are very rare accounting for less than $0.1 \%$ of all malignancies. We report a rare clinical image of advanced stage soft tissue sarcoma of the scalp. A 65 year woman had presented to the surgical department with complaints of a rapidly growing swelling over the scalp for three months. On examination there was huge $20 \times 20 \mathrm{~cm}$ swelling over the scalp in the left temporoparietal region with variegated consistency. Computed tomography of head revealed a large soft tissue mass with necrosis invading the bone and underlying brain parenchyma. Histopathological finding from core needle biopsy revealed pleomorphic sarcoma. STS are highly malignant tumors which should be diagnosed and treated using multimodality approach. Recurrences are common even after complete resection and prognosis is poor.

\section{KEYWORDS}

Scalp tumor, Soft tissue tumor, Intracerebral invasion, Pleomorphic sarcoma, Giant scalp tumor

Received on: May 22, 2021

Accepted for publication: August 23, 2021

\section{CORRESPONDING AUTHOR}

Dr. Raghav Yelamanchi, Junior Consultant,

Department of Surgery, Atal Bihari Vajpayee Institute of Medical Sciences and Dr. Ram Manohar Lohia Hospital, New Delhi, India, Pin-110001

Email: raghavyelamanchi@gmail.com

Orcid No: https://orcid.org/0000-0001-6786-8056

DOI: https://doi.org/10.3126/nmcj.v23i4.42269 


\section{CASE PRESENTATION}

A 65 year old woman had presented to the surgical department with complaints of a rapidly growing swelling over the scalp for three months. There was history of occasional bleeding from the swelling. On examination there was huge $20 \times 20 \mathrm{~cm}$ swelling over the scalp in the left temporoparietal region with variegated consistency [Figure 1]. The swelling was fixed to the underlying bone. There were no neurological deficits. Computerized tomography of head revealed a large soft tissue mass with necrosis invading the bone and underlying brain parenchyma [Figure 2]. Histopathological finding from core needle biopsyperformedunderimageguidanceshowed spindle cells with moderate pleomorphism and hyperchromatic nuclei with eosinophilic cytoplasm. Immunohistochemistry was suggestive of pleomorphic sarcoma. Further imaging of the chest and abdomen revealed no metastasis.

Even locally advanced scalp tumors can be resected with reasonable outcomes. Invasion of skull bone and limited invasion of brain parenchyma can also be resected with ease. However, the large defect created after resection requires closure using suitable flap. In the above case, the invasion of the brain parenchyma was extensive and the performance status of the patient was not adequate to tolerate such a morbid procedure. The case was discussed in tumor board and was advised palliative chemotherapy and radiotherapy considering the locally advanced unresectable disease and performance status of the patient. Patient is ambulatory at two months of follow-up while on palliative therapy. However, she has decreased quality of life due to the tumor mass and its effects.

\section{DISCUSSION}

Soft tissue sarcomas (STS) most commonly occur in the extremities and retroperitoneum. Scalp soft tissue tumors are very rare accounting for less than $0.1 \%$ of all malignancies. The exact etiology of soft tissue sarcomas of scalp is not elucidated. ${ }^{1}$ These tumors are more common in the elderly population and chronic exposure to ultraviolet radiation from sunlight is hypothesized as a possible etiology. ${ }^{2}$ Other risk factors include xeroderma pigmentosum and immunosuppression. ${ }^{2}$ STS are very aggressive tumors. They grow rapidly and invade the underlying structures. They spread by hematogenous route and cause distant metastasis. $^{3}$
The periosteum of the skull will act as a natural barrier to the spread of scalp tumors. ${ }^{4}$ But, in very rare cases where tumor grows to a very large size, it can invade the underlying bony cortex and also the brain parenchyma. As the STS grow rapidly they undergo significant necrosis. Biopsy under image guidance is required to sample the solid region and avoid sampling errors. Pleomorphic sarcoma of scalp is a challenging diagnosis. Histologically, differentiation from its benign counterpart is difficult as both have similar histopathological features of spindle cells and CD 10 positivity. ${ }^{5}$ Differentiation of benign and malignant tumors should be done clinically, radiologically and by histopathology. Clinical and imaging findings are helpful in cases of small tumors with equivocal pathological findings. ${ }^{6}$ Giant tumors as the case mentioned above are typically malignant and present no doubts.

The treatment of these large tumors is challenging. Wide local excision is the treatment of choice but the difficulty in case of scalp tumors is the defect closure which requires tissue flaps. ${ }^{7}$ Even after transfer, it is not cosmetically aesthetic due to the lack of hair over the transplanted portion. ${ }^{7}$ In case of bony and cerebral invasion, these tumors can present with emergencies like intracerebral or extracerebral bleed. Limited invasion of the brain parenchyma can be treated with excision but large lesions are often unresectable and are associated with morbidity. Even if they are resected with negative margins they are associated with a high recurrence rate of around $20 \%{ }^{8}$ There are no specific treatment guidelines for STS of scalp. Adjuvant radiation has not shown to reduce the rate of recurrence.

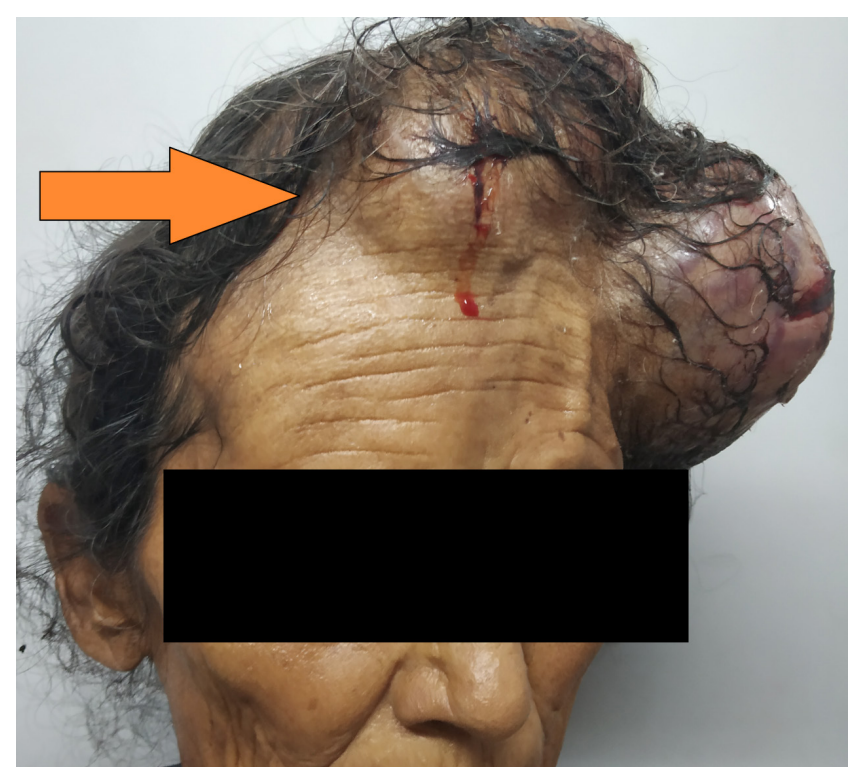

Fig. 1: Soft tissue tumor of the scalp 


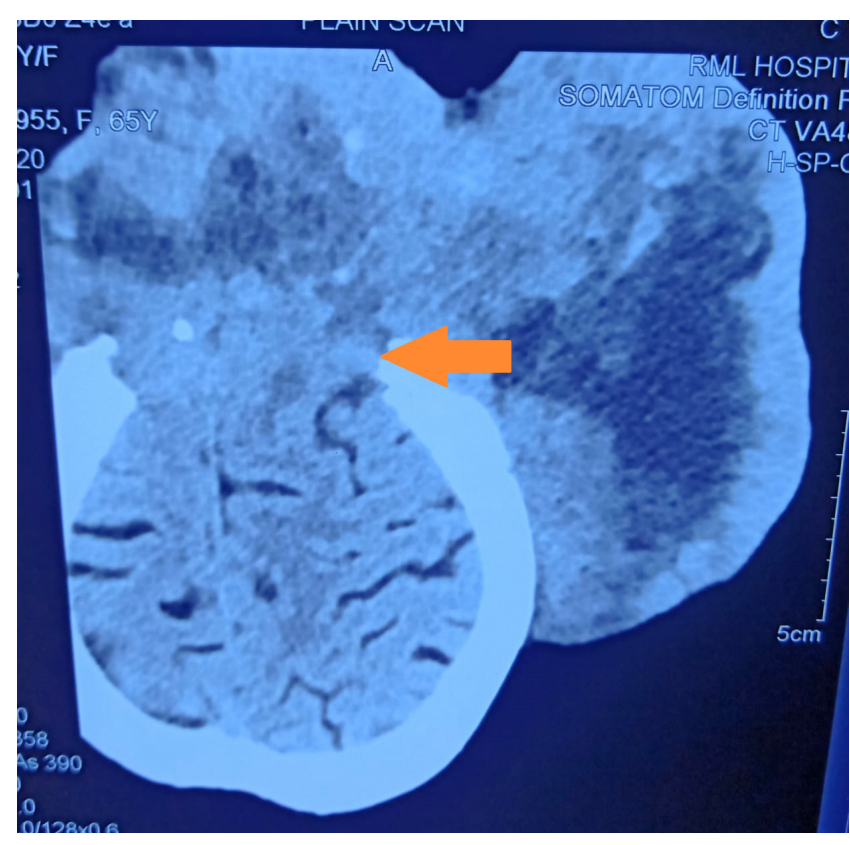

Fig. 2: Computed tomography of the head showing soft tissue mass invading the skull and brain parenchyma
Palliative chemotherapy and radiotherapy are the only options for locally unresectable or metastatic disease.

STS are highly malignant tumors which should be diagnosed and treated using multimodality approach. Recurrences are common even after complete resection and prognosis is poor.

\section{Conflict of interests: None}

\section{Source of research fund: None}

Ethical approval: All procedures performed in studies involving human participants were in accordance with the ethical standards of the institutional and/or national research committee and with the 1964 Helsinki declaration and its later amendments or comparable ethical standards.

Informed consent: Informed consent was obtained from all individual participants included in the study.

\section{REFERENCES}

1. Sturgis EM, Potter BO. Sarcomas of the head and neck region. Curr Opin Oncol 2003; 15: 239-52. doi: 10.1097/00001622-200305000-00011.

2. Klebanov N, Hoang MP, Reddy BY. Pleomorphic Dermal Sarcoma of the Scalp. Cureus 2018; 10: e2979. doi: 10.7759/cureus.2979.

3. Casali PG, Abecassis N, Aro HT et al. ESMO Guidelines Committee and EURACAN. Soft tissue and visceral sarcomas: ESMO-EURACAN Clinical Practice Guidelines for diagnosis, treatment and follow-up. Ann Oncol. 2018;29(Suppl 4):iv51iv67. doi: 10.1093/annonc/mdy096.

4. Lin PP, Pino ED, Normand AN et al. Periosteal margin in soft-tissue sarcoma. Cancer 2007; 109: 598-602. doi: 10.1002/cncr.22429.

5. Wollina U, Schönlebe J, Bujok V et al. Dermal Pleomorphic Sarcoma of the Scalp - Report of Two Cases. Open Access Maced J Med Sci 2019; 7: 2982-4. doi: 10.3889/oamjms.2019.274.
6. Tran NA, Guenette JP, Jagannathan J. Soft Tissue Special Issue: Imaging of Bone and Soft Tissue Sarcomas in the Head and Neck. Head Neck Pathol 2020; 14: 132-43. doi: 10.1007/s12105-01901102-5.

7. Baldelli I, Mangialardi ML, Salgarello M, Raposio E. Surgical Reconstruction following Wide Local Excision of Malignant Melanoma of the Scalp. Plast Reconstr Surg Glob Open 2020; 8: e3059. doi: 10.1097/GOX.0000000000003059.

8. Jasper KD, Holloway CL, DeVries KJ, Truong PT. Local Relapse and Survival Outcomes in Patients with Scalp Sarcoma: A Retrospective Study of 95 Patients Treated in a Provincial Cancer Care Institution Over 25 Years. Cureus 2019; 11: e5236. doi: 10.7759/cureus.5236. 